\title{
Aa. Vv., Gustave Kahn (1859-1936). Études réunies par Sophie Basch
}

Ida Merello

\section{OpenEdition}

1 Journals

\section{Edizione digitale}

URL: http://journals.openedition.org/studifrancesi/6392

DOI: 10.4000/studifrancesi.6392

ISSN: 2421-5856

\section{Editore}

Rosenberg \& Sellier

\section{Edizione cartacea}

Data di pubblicazione: 1 novembre 2010

Paginazione: 579

ISSN: 0039-2944

\section{Notizia bibliografica digitale}

Ida Merello, «Aa. VV., Gustave Kahn (1859-1936). Études réunies par Sophie Basch», Studi Francesi [Online], 162 (LIV | III) | 2010, online dal 30 novembre 2015, consultato il 09 janvier 2021. URL: http:// journals.openedition.org/studifrancesi/6392 ; DOI: https://doi.org/10.4000/studifrancesi.6392

Questo documento è stato generato automaticamente il 9 janvier 2021.

\section{(c) (i) (9)}

Studi Francesi è distribuita con Licenza Creative Commons Attribuzione - Non commerciale - Non opere derivate 4.0 Internazionale. 


\title{
Aa. Vv., Gustave Kahn (1859-1936). Études réunies par Sophie Basch
}

\author{
Ida Merello
}

\section{NOTIZIA}

Aa. Vv., Gustave Kahn (1859-1936). Études réunies par Sophie Basch, Paris, Classiques Garnier, 2009, pp. 505.

1 Per l'alto livello di tutti i contributi il volume rappresenta un momento importante della storia della critica non solo riguardo agli studi su Kahn, ma anche, più in generale, sulla poesia fin de siècle. Diviso in tre parti, dedica la prima agli studi sulla poetica e la lingua dell'A., la seconda alla sua attività di critico letterario e artistico, la terza alle sue amicizie letterarie. Così nella prima parte Pascal DURAND (Le Théâtre du pauvre: de Mallarmé à K., pp.11-21) propone alcune riflessioni sull'articolo di Kahn Un théâtre de l'avenir apparso nel 1889 sulla «Revue d'art dramatique». L'A. attribuisce l'interesse di Kahn per i clown non tanto a una proiezione dell'estraneità al mondo propria dell'ambiente simbolista decadente, quanto a una nostalgia degli elementi popolari, percepiti come espressione di un'energia vitale primitiva. Mostra così in tal modo come la teoria di Kahn sul teatro sia anticipatrice dell'estetica modernista. sophie BASCH (Théâtre de l'avenir, mannequins du présent et nostalgie du guignol, pp. 23-42) parte dal medesimo scritto di Kahn, sottolineando a sua volta come l'interesse per i clowns e la pantomima rappresenti un'esigenza di autenticità nella piena consapevolezza che essa è contraddetta ogni volta che il testo clownesco viene redatto in veste autoriale: l'A. prende in esame quindi gli altri testi che Kahn ha dedicato all'argomento, individuando i rapporti con le arti figurative, la riflessione su Guignol e quegli elementi che annunciano l'estetica popolare delle prime pellicole cinematografiche. Evanghelia STEAD (Soixante et un mots de G. K. recueillis par Jacques Plowert, pp. 45-65) approfondisce il suo lavoro di lunga data sul Petit glossaire pour servir à l'intelligence des auteurs décadents et symbolistes, individuando la componente attribuibile a Kahn nella redazione dell'opera 
uscita sotto lo pseudonimo a largo spettro di Plowert. L'A. ritiene infatti probabile che l'abbondante quantità di parole (sessantuno) tratte da testi di Kahn sia stata commentata dall'autore stesso, e mostra come l'innovazione linguistica dei termini che compaiono anche nel Littré si realizzi attraverso l'ampliamento della gamma dei significati; mentre, per quanto riguarda gli altri termini, a dominare sono la trasgressione grammaticale e l'evocazione suggestiva. Michel MURAT (L'initiateur du vers libre, pp. 67-91) analizza l'incidenza degli scritti di Kahn presso i suoi contemporanei, offrendone la definizione di iniziatore del verslibrisme piuttosto che del verso libero, per natura polimorfo. L'A. mostra come si debba a Kahn lo stabilirsi di quel legame tra verso libero e movimento simbolista che comincerà a essere messo in discussione solo dopo la curva del secolo e delinea esemplarmente le diverse vie al fenomeno; in seguito si sofferma sull'analisi della strofa di Kahn, e sull'identificazione delle caratteristiche ritmiche del verso di Kahn confrontato con quello di altri poeti a lui contemporanei; sottolinea infine l'anticipazione in Kahn di alcuni elementi costitutivi del verso modernista, come l'abolizione delle maiuscole a inizio verso e del punto finale della frase. Anne Holmes (K., Laforgue et la question du vers libre, pp. 93-109) segue l'amicizia poetica di Laforgue e di Kahn, mostrando l'ingenerosità del secondo per il mancato apprezzamento del ruolo poetico giocato dal primo. Jean-Luc STEINMETZ (G.K. et Mallarmé, pp.111-121) ripercorre il rapporto con Mallarmé, mostrando l'opposizione tra la libertà invocata da Kahn e la massima sperimentazione all'interno dell'alessandrino di Mallarmé; con un'attenzione al verso libero da parte di quest'ultimo condizionata dalla modesta qualità di Kahn poeta. Henri SCEPI (G.K.: vers libre et position lyrique, pp. 123-142) ricorda come nel dibattito sul verso libero l'oralità abbia costituito per taluni un principio costitutivo del verso non metrico, definisce a sua volta il verso libero come «trace segmenté, variable, incessamment retravaillé parce que toujours recommencé, d'un phrasé» e riconosce il merito a Kahn di aver mostrato il legame indissolubile tra soggetto parlante, movimento simultaneo del discorso e del verso, forma generale della poesia. Apre la seconda parte Jean DE PALACIO (G.K. et l'Ecclésiaste, pp. 146-157) con una riflessione sul'opera poetica di K. influenzata dalla Bibbia, e in particolare il Prélude à une paraphrase de l'Ecclésiaste di cui mostra, in rapporto anche col Fragment d'un commentaire sur l'Ecclésiaste di Mendès, i tratti distintivi di K., individuati in un'apertura verso la natura e un sentimento decadente della marcescenza. Hélène VÉDRINE (Variations shakespeariennes, en vers et en prose, pp.159-191) analizza l'attenzione di Kahn verso Shakespeare, di cui coniuga i personaggi di Amleto e Lear (intesi come rappresentazione della follia del poeta e dell'uomo di potere) in Le Conte de l'or et $d u$ silence e Le Roi fou, nell'ambito di un'attenzione politica che l'A. gli riconosce come precipua. In filigrana l'A. riconosce tuttavia come più prossimo $\mathrm{a} \mathrm{K}$. il testo delle Moralités légendaires di Laforgue À propos d'Hamlet. Roland MORTIER (G.K. et le XVIII siècle français, pp.193-208) presenta invece il côté dix-huitiémiste di Kahn, studioso di Casanova, attento a Diderot in un'epoca più interessata a Voltaire e a Rousseau, nonché esperto misconosciuto di pittura rococò. André GUYAUX (G.K. lecteur de Baudelaire, pp. 209-218) si sofferma sull'attenzione di Kahn nei confronti di Baudelaire inteso come ispiratore del verso libero, riconoscendovi una manifestazione di quell'«école Baudelaire» di cui les Taches d'encre di Barrès rappresentarono la prima messa a fuoco storica e all'interno della quale lo stesso Kahn distingueva i cattivi discepoli (néo parnassiens) dai buoni, ossia i simbolisti. Per BuviK (Ibsen et Bjørnson selon G.K., pp. 219-231) ci rivela invece l'interesse di K. per le letterature scandinave, con particolare attenzione a Ibsen non solo per la produzione teatrale, ma anche quella poetica. Fabrice wiLHELM (Le rêve, roman 
du demi-mensonge, pp. 233-249) offre una lettura attenta del romanzo Le Rêve di Zola, di cui coglie bene anche le intenzioni meno evidenti, e mostra l'evoluzione dell'atteggiamento di Kahn nei confronti dello scrittore a seguito dell'impegno nell'affaire Dreyfus. Ségolène LE MEN (L'introduction de G.K. aux dessins de Georges Seurat: une biographie en pointillé, pp. 251-304) propone un vasto saggio che investe i rapporti tra K. e Seurat attraverso un'accurata disamina degli interventi critici di K. e in particolare della sua prefazione al catalogo dei disegni di Seurat, prefazione che viene indagata sia dal punto di vista del metodo critico che del suo rilievo storiografico. Laurence BROGNIEZ (K. et les préraphaélites: la critique d'art comme laboratoire du symbolisme, pp. 305-322) mostra come i poeti simbolisti francesi si siano appropriati del movimento preraffaellita, e mette in evidenza la specificità della critica di K. posta in relazione con quella dei critici contemporanei francesi e belgi. Colette CAMELIN ( $L$ 'intense travail mental de l'artiste». G.K. et Paul Gauguin, pp. 323-342) sofferma la sua attenzione sul lungo saggio dedicato da K. a Gauguin, che apprezzava come pittore maledetto e intellettuale puro. La terza parte, dedicata al «réseau littéraire» si apre con il saggio di Jean-Pierre BERTRAND (De l'amitié littéraire: Laforgue et K. ou la naissance d'une clique, pp. 343-372) che studia i meccanismi dell'amicizia di K. con Laforgue, fondata su di un'implicita concorrenza e sull'appartenenza a una clique, ossia una rete comune di complicità intellettuale. Bertrand MARCHAL offre una lettera inedita di Mallarmé a G.K. (pp. 373-374); Adrienne fonTAINAS (Les éditeurs belges de G.K.: Paul Lacomblez, la veuve Monnom et Edmond Deman, pp.375-394) misura i rapporti di Kahn con gli editori belgi di cui mette in luce le politiche editoriali. Così emerge il ruolo di primo piano dell'editore Deman, ugualmente attento all'avanguardia poetica che a quella pittorica, che finanzia una splendida edizione illustrata da Lemen dei Limbes de lumière. Fabrice VAN DE KERCKHOVE, Du rejet à l'adoption du symbolisme: «L'Art moderne» et «La Wallonie», pp.395-457) prosegue l'analisi della recezione belga di K., mostrando l'influenza di Verhaeren e di Khnopff sul direttore dell'«Art moderne», piegato al simbolismo e aperto agli scambi con le riviste francesi. Ma l'A. segue con analogo interesse anche i legami con «La Wallonie» «La Jeune Belgique» e «La Société nouvelle». Liana NISSIM (G.K. et la revue «Poesia» de Filippo Tommaso Marinetti, pp.459-482) vede i due autori accomunati da un medesimo pregiudizio critico che li ha relegati in un ruolo di minori cui Marinetti solo recentemente è stato sottratto, mettendo in luce la perfidia nei confronti di entrambi sia di Claudel che di Gide. L'A. si sofferma quindi sul rapporto tra i due autori nelle pubblicazioni della rivista «Poesia». Marie-Brunette SPIRE (G.K. et la revue «Menorah», pp. 483-506) ricorda come l'appartenenza alla razza ebraica sia stata importante per K. che dal 1923 al 1933 dirige la rivista militante sionista «Menorah»e pubblica raccolte che hanno soggetti ebraici; mostra infine il suo rifiuto di far coincidere il sionismo con la volontà di costituire una nazione ebraica, per auspicare invece un'integrazione nei diversi Paesi. Ma l'A. pone altresì l'accento sull'evoluzione del pensiero di K., che il pregiudizio razziale dell'epoca rende sempre più consapevole di un'appartenenza a una tradizione e a una cultura ebraiche. 